\title{
Influence of compression-expansion effect on oscillating-flow heat transfer in a finned heat exchanger*
}

\author{
Ke TANG, Juan YU, Tao JIN ${ }^{\dagger \ddagger}$, Zhi-hua GAN \\ (Institute of Refrigeration and Cryogenics, Zhejiang University, Hangzhou 310027, China) \\ †E-mail: jintao@zju.edu.cn \\ Received Mar. 11, 2013; Revision accepted Apr. 19, 2013; Crosschecked May 16, 2013
}

\begin{abstract}
Compression and expansion of a working gas due to the pressure oscillation of an oscillating flow can lead to a temperature variation of the working gas, which will affect the heat transfer in the oscillating flow. This study focuses on the impact of the compression-expansion effect, indicated by the pressure ratio, on the heat transfer in a finned heat exchanger under practical operating conditions of the ambient-temperature heat exchangers in Stirling-type pulse tube refrigerators. The experimental results summarized as the Nusselt number are presented for analysis. An increase in the pressure ratio can result in a marked rise in the Nusselt number, which indicates that the compression-expansion effect should be considered in characterizing the heat transfer of the oscillating flow, especially in the cases with a higher Valensi number and a lower maximum Reynolds number.
\end{abstract}

Key words: Heat transfer, Heat exchanger, Oscillating flow, Pressure ratio, Pulse tube refrigerator doi:10.1631/jzus.A1300076

Document code: A

CLC number: TK124

\section{Introduction}

A pulse tube refrigerator, one of the typical regenerative cryocoolers, employs an oscillating flow to realize cryogenic refrigeration (de Waele, 2011). The oscillating flow is characterized by the cyclically reversion of flow direction, which leads to distinct features of the flow and the heat transfer, in contrast with the unidirectional steady flow. Although much effort (Swift, 1992; 2002; Cooper et al., 1994; Zhao and Cheng, 1996; Gusev et al., 2001; Bouvier et al., 2005; Nsofor et al., 2007; Tang et al., 2007; 2011; Meng et al., 2008; Chen et al., 2009; Shi et al., 2010; Piccolo, 2011; de Waele, 2012; Jaworski and Piccolo, 2012) has been made on numerical simulation and experimental observation of the heat transfer in an oscillating flow, it is still a big challenge for accu-

\footnotetext{
\$Corresponding author

* Project (No. 51176167) supported by the National Natural Science Foundation of China

(C) Zhejiang University and Springer-Verlag Berlin Heidelberg 2013
}

rately designing the heat exchanger for a pulse tube refrigerator, due to the complexity of the oscillating flow.

Compression and expansion of a working gas due to the pressure oscillation of oscillating flow can lead to a variation in temperature of the working gas, which will influence the heat transfer between the oscillating flow and the solid wall. However, there is, so far, a lack of the knowledge about the impact of the compression-expansion effect on the heat transfer in oscillating flow, and this impact is seldom considered in the reported work. Swift (1992) and Piccolo (2011) discussed the influence of the drive ratio (the ratio of pressure amplitude to mean working pressure) on the heat transfer in the oscillating flow; however, the relative fluid displacement amplitude $\left(A_{\mathrm{r}}=2 x_{\mathrm{A}} / L=\right.$ $2 u_{\mathrm{A}} /(\omega L)$, where $2 x_{\mathrm{A}}$ is the peak-to-peak displacement of the working gas, $u_{\mathrm{A}}$ is the velocity amplitude, $\omega$ is the angular frequency, and $L$ is the length of the heat exchanger) was changed with the varying drive ratio in their experiments and simulations. Their discussion concerned the impact of the relative fluid displace- 
ment amplitude $A_{\mathrm{r}}$, instead of the compressionexpansion effect.

The compression-expansion effect can be indicated by the pressure ratio, which is usually defined as $\left(p_{\mathrm{m}}+p_{\mathrm{A}}\right) /\left(p_{\mathrm{m}}-p_{\mathrm{A}}\right)$ in the field of cryocoolers (where $p_{\mathrm{m}}$ is the mean working pressure, and $p_{\mathrm{A}}$ is the pressure amplitude). The present work focuses on the impact of the compression-expansion effect on the heat transfer in the oscillating flow via the pressure ratio. An experimental apparatus was built to observe the space-time averaged heat transfer characteristics of a finned heat exchanger. The experimental operating conditions were set according to the ambienttemperature heat exchangers of Stirling-type pulse tube refrigerators (Dietrich and Thummes, 2010; Qiu et al., 2012). The ambient-temperature heat exchangers are used to remove the heat from the warm working gas to the surroundings, and an insufficient heat transfer of the ambient-temperature heat exchangers could deteriorate the refrigeration performance of the pulse tube refrigerators. After a description of the experimental apparatus including the measurement system, the dimensionless similarity criteria used in this study are introduced. The experimental results are summarized into Nusselt number. The emphasis will be put on the dependence of the Nusselt number on the pressure ratio.

\section{Experimental}

Fig. 1 shows the schematic of the experimental apparatus, consisting of a linear compressor, a test heat exchanger, a needle valve, and a reservoir.

The linear compressor, which is usually used to drive a Stirling cooler or a Stirling-type pulse tube refrigerator, is the generator of the oscillating flow. The K535 compressor fabricated by Ricor and the $2 \mathrm{~S} 102 \mathrm{~W}$ compressor fabricated by QDrive were used to realize diverse operating conditions. To ensure safe operation, the working current and voltage of the K535 compressor were restricted below $10 \mathrm{~A}$ and $50 \mathrm{~V}$, respectively, and those of the $2 \mathrm{~S} 102 \mathrm{~W}$ compressor were controlled below $4 \mathrm{~A}$ and $110 \mathrm{~V}$, respectively. The compressor was powered by an $\mathrm{AC}$ power supply of Type-PCR1000LA, fabricated by the Kikusui Electronics Co., with the operating parameters of AC 1-150 V/2-300 V, $1 \mathrm{kVA}$, and 1-999.9 Hz.

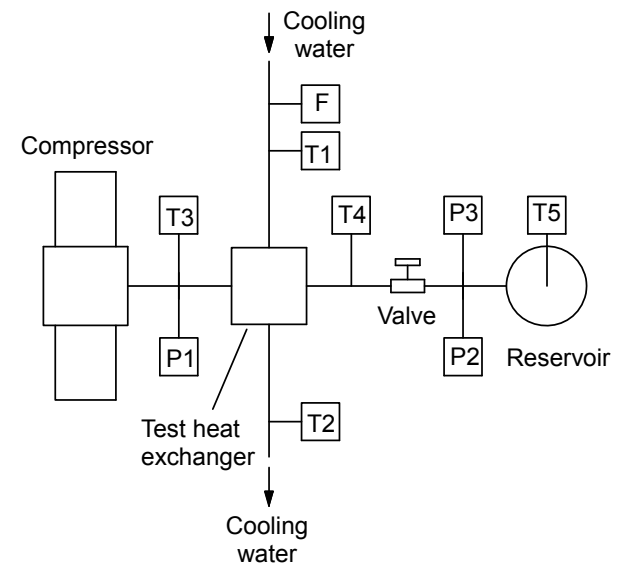

Fig. 1 Schematic of the experimental apparatus $\mathrm{T} 1, \mathrm{~T} 2, \mathrm{~T} 3, \mathrm{~T} 4$, and T5 denote the thermometers, P1, P2, and $\mathrm{P} 3$ denote the pressure sensors, and $\mathrm{F}$ denotes the flowmeter

The test heat exchanger, made of copper, has a finned configuration as shown in Fig. 2. The fin thickness and the fin gap are both $0.75 \mathrm{~mm}$. The fin heights range from $2.6 \mathrm{~mm}$ to $13.6 \mathrm{~mm}$, with a uniform length of $20 \mathrm{~mm}$ (i.e., the length of the test heat exchanger is $20 \mathrm{~mm}$ ). The heat exchanger was cooled by water, which was supplied by a thermostat set at $293 \mathrm{~K}$. The heat exchanger, together with the tubes for the working gas and the cooling water flowing into and out of the heat exchanger, was thermally isolated by polystyrene foam to reduce the influence of heat transfer with the surroundings.
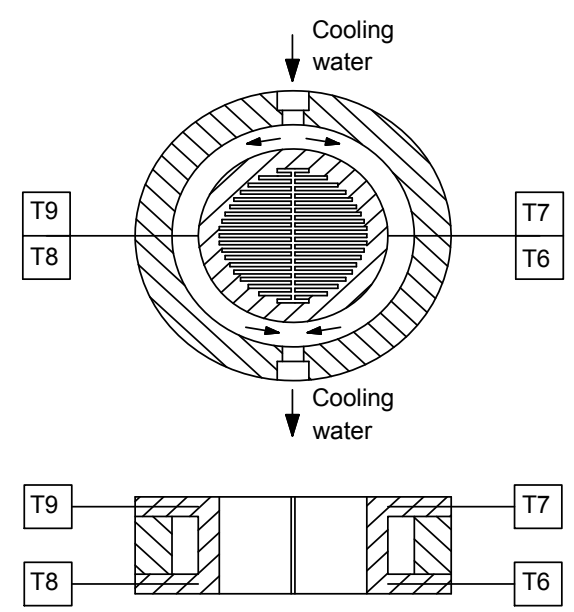

Fig. 2 Schematic of the finned heat exchanger

For the oscillating flow, a needle valve and a reservoir can form a resistance-compliance impedance, which is usually employed in thermoacoustic 
engines to measure the acoustic power output (Tang et al., 2008). In this work, the valve-reservoir configuration was used to measure the velocity amplitude of the oscillating flow.

The measured parameters include temperature, pressure, and flow rate, as shown in Figs. 1 and 2.

Two platinum resistance thermometers (PT100) of $\mathrm{T} 1$ and $\mathrm{T} 2$ were used to measure the temperatures of the cooling water flowing into and out of the heat exchanger, by inserting the thermometers into the cooling water through two tee-fittings. The temperatures of working gas at the two ends of the heat exchanger, as well as the working gas temperature in the reservoir, were measured by three PT100 thermometers of T3, T4, and T5 with $2 \mathrm{~mm}$ in diameter and $7 \mathrm{~mm}$ in length. T3, T4, and T5 were put in the high pressure working gas, and the electricity power supply and voltage signals were transmitted via electric connectors. Additionally, two cone chambers were set to join the ends of the heat exchanger and the connecting tubes. To measure the average temperature of the working gas flowing into and out of all passages formed by the fins, the thermometers of $\mathrm{T} 3$ and $\mathrm{T} 4$ were located in the cone chambers. Four K-type sheathed thermocouples of T6, T7, T8, and $\mathrm{T} 9$, with $0.5 \mathrm{~mm}$ in diameter, were inserted into the flange of the heat exchanger to measure the wall temperature. Considering the high sensitivity to the temperature differences instead of the absolute temperatures in this measurement, all these nine thermometers were simultaneously calibrated in a variable-temperature water bath to correct the temperature measurement to $\pm 0.1 \mathrm{~K}$.

The pressures, $\mathrm{P} 1$ and $\mathrm{P} 2$, were measured by two pressure sensors with the range of $0-5 \mathrm{MPa}$ and the accuracy of $0.2 \%$ FS $(10 \mathrm{kPa})$. A dynamic pressure sensor $\mathrm{P} 3$ with an accuracy of $0.57 \mathrm{kPa}$ was used for more accurate measurement of the dynamic pressure in the reservoir. The pressure amplitude $p_{\mathrm{A} 3}$ in the reservoir is a key parameter for calculating the velocity amplitude $u_{\mathrm{A}}$ in the test heat exchanger, and then with a known $u_{\mathrm{A}}$, the maximum Reynolds number $R e_{\max }$, the Mach number $M a$, and the relative fluid displacement amplitude $A_{\mathrm{r}}$ can all be calculated. More details of the related formulae will be given in the next section.

Besides the temperature and pressure measurement, a mini turbine flowmeter with an accuracy of
$0.005 \mathrm{~L} / \mathrm{min}$ was used for the measurement of the cooling water.

Additionally, as shown in Fig. 1, we do not need a hot heat exchanger in our experimental apparatus to heat the working gas. Actually, the working gas out from the linear compressor was heated by the Joule heating effect due to the current and resistance of coils in the compressor, and the working gas out from the valve-and-reservoir configuration was heated due to the viscous dissipation in the small flow channel of the needle valve.

\section{Dimensionless similarity criterion}

Dimensionless similarity criteria were used in this study to summarize the experimental data. Five dimensionless similarity criteria, i.e., the maximum Reynolds number $R e_{\max }$, the Valensi number $V a$, the Prandtl number $P r$, the specific heat ratio $\gamma$, and the Mach number $M a$, were determined according to the governing equations (Anderson, 1995) for the unsteady flow of compressible viscous fluid (Appendix). The Prandtl number and specific heat ratio are the thermophysical properties of working fluid. The definitions of $R e_{\max }, V a$, and $M a$ are as follows:

$$
\begin{aligned}
& R e_{\max }=\frac{u_{\mathrm{A}} D_{\mathrm{h}}}{v}, \\
& V a=\frac{\omega D_{\mathrm{h}}^{2}}{v}, \\
& M a=\frac{u_{\mathrm{A}}}{a},
\end{aligned}
$$

where $u_{\mathrm{A}}$ is the velocity amplitude, $D_{\mathrm{h}}$ is the hydraulic diameter, $\omega$ is the angular frequency, $v$ is the kinematic viscosity, and $a$ is the sound speed. According to the pressure amplitude $p_{\mathrm{A} 3}$ in the reservoir measured by sensor $\mathrm{P} 3$, the velocity amplitude $u_{\mathrm{A}}$ can be calculated by (Tang et al., 2008):

$$
u_{\mathrm{A}}=\frac{U_{\mathrm{A}}}{A_{\text {flow }}}=\frac{p_{\mathrm{A} 3} \omega C_{\mathrm{res}}}{A_{\text {flow }}}=\frac{2 \pi f p_{\mathrm{A} 3} V_{\mathrm{res}}}{A_{\text {flow }} \gamma p_{\mathrm{m} 2}}
$$

where $U_{\mathrm{A}}$ is the volume velocity amplitude, $A_{\text {flow }}$ is the flow area in the test heat exchanger, $C_{\text {res }}$ is the compliance of the reservoir, $f$ is the frequency, $V_{\text {res }}$ is the 
volume of the reservoir, and $p_{\mathrm{m} 2}$ is the mean working pressure in the reservoir measured by sensor P2.

As previously mentioned, the peak-to-peak displacement of the working fluid with respect to the length of the heat exchanger is an important factor for the heat transfer in oscillating flow (Swift, 1992; 2002; Piccolo, 2011). The dimensionless parameter of the relative fluid displacement amplitude $A_{\mathrm{r}}$ was also considered in this study.

The space-time averaged heat transfer performance is presented by the Nusselt number, which is defined as

$$
\begin{gathered}
N u=\frac{h D_{\mathrm{h}}}{k}, \\
h=\frac{Q_{\mathrm{h}}}{\left(A_{1}+\sum_{i=1}^{n} A_{2, i} \eta_{\mathrm{fin}, i}\right)\left(t_{\mathrm{f}}-t_{\mathrm{wi}}\right)},
\end{gathered}
$$

where $k$ is the thermal conductivity of the working fluid, $h$ is the convective heat transfer coefficient, $Q_{\mathrm{h}}$ is the heat flow transferred from the oscillating flow, $A_{1}$ is the area of the base surface, $A_{2, i}$ and $\eta_{\text {fin }, i}$ are the surface area and the fin efficiency of the $i$ th fin, respectively, $n$ is the number of the fins, and $t_{\mathrm{f}}$ and $t_{\mathrm{wi}}$ are the temperatures of the oscillating flow and the base surface, respectively.

$Q_{\mathrm{h}}$ was estimated according to the heat flow $Q_{\mathrm{c}}$ of the cooling water by considering the heat transfer with the surroundings based on a thermal balance analysis. $Q_{\text {c }}$ can be calculated by

$$
Q_{\mathrm{c}}=q_{\mathrm{v}} \rho_{\text {water }} c_{\text {water }}\left(t_{2}-t_{1}\right),
$$

where $q_{\mathrm{V}}$ is the volume flow rate of the cooling water measured by the flowmeter F (Fig. 1), $\rho_{\text {water }}$ and $c_{\text {water }}$ are the density and the specific heat of the cooling water, $t_{1}$ and $t_{2}$ are the temperatures of the cooling water flowing into and out of the test heat exchanger measured by the thermometers $\mathrm{T} 1$ and $\mathrm{T} 2$, respectively (Fig. 1).

Additionally, the average temperature of T3 and T4 is taken as the temperature of the oscillating flow $t_{\mathrm{f}}$ The wall temperature $t_{\mathrm{wi}}$ is determined according to thermometers T6, T7, T8, and T9, considering the thermal conduction inside the copper wall. The fin efficiency $\eta_{\text {fin, } i}$ is calculated with the typical formulae for the rectangular fin (Incropera et al., 2007).

\section{Results and analysis}

With helium as the working gas, the heat transfer performance of the finned heat exchanger was tested under a mean working pressure of 2.0 MPa with the K535 compressor, and was also tested under a higher mean working pressure of $2.5 \mathrm{MPa}$ with the $2 \mathrm{~S} 102 \mathrm{~W}$ compressor. The operating frequency ranged from $45 \mathrm{~Hz}$ to $100 \mathrm{~Hz}$. According to the critical dimensionless value $\left(\beta_{\text {cri }}=2 R e_{\max } / V a^{0.5}\right)$ of 400-780 (Merkli and Thomann, 1975; Hino et al., 1976), all the oscillating flow cases in our experiments were in the range of laminar flow.

Table 1 tabulates the typical experimental data under the mean working pressure of $2.0 \mathrm{MPa} . p_{\mathrm{m} 1}$ and $\mathrm{PR}_{1}$ denote the mean working pressure and the pressure ratio at P1 (Fig. 1), respectively. Since the mean working pressure was kept almost constant and the variation of working gas temperature $t_{\mathrm{f}}$ was small (less than $10 \mathrm{~K}$ ), the Prandtl number and the specific heat ratio, calculated by REFPROP 8.0, are constant. By adjusting the voltage and the frequency of the $\mathrm{AC}$ power supply, as well as the opening of the needle valve, the dependence of Nusselt number on pressure ratio was tested with the maximum Reynolds number, and the Valensi number almost fixed. According to Eqs. (1) and (3), the fixed maximum Reynolds number implies an almost constant Mach number, due to the tiny variation of the sound speed in our test. Moreover, for the test heat exchanger, the almost fixed velocity amplitude and angular frequency implied by the maximum Reynolds number and Valensi number (Eqs. (1) and (2)) lead to roughly unchanged relative fluid displacement amplitude.

Table 1 shows that an increase of pressure ratio from 1.100 to 1.300 results in a rise of Nusselt number from 2.694 to 5.395 , roughly by $100 \%$. The marked rise of Nusselt number with the pressure ratio, while keeping other dimensionless similarity criteria constant, indicates that the pressure ratio is an independent factor needing to be considered for the heat transfer of the oscillating flow.

Additionally, a stronger compression-expansion effect, indicated by a larger pressure ratio, can enhance the heat transfer of the oscillating flow shown by a larger Nusselt number. This may be attributed to the disturbance of the thermal boundary layer. A higher pressure ratio operation can lead to a stronger 
Table 1 Typical experimental data under mean working pressure of 2.0 MPa

\begin{tabular}{ccccccccc}
\hline$p_{\mathrm{m} 1}$ & $\mathrm{PR}_{1}$ & $R e_{\max }$ & $V a$ & $P r$ & $c_{\mathrm{p}} / c_{\mathrm{v}}$ & $M a$ & $A r$ & $N u$ \\
\hline 1.996 & 1.300 & 200.6 & 99.7 & 0.660 & 1.664 & 0.0135 & 0.302 & 5.395 \\
2.000 & 1.261 & 200.0 & 100.4 & 0.660 & 1.664 & 0.0133 & 0.299 & 4.965 \\
2.016 & 1.219 & 199.9 & 100.6 & 0.660 & 1.664 & 0.0131 & 0.298 & 4.429 \\
2.008 & 1.180 & 200.6 & 100.3 & 0.660 & 1.664 & 0.0133 & 0.300 & 3.621 \\
1.999 & 1.140 & 200.2 & 100.1 & 0.660 & 1.664 & 0.0133 & 0.300 & 3.196 \\
1.999 & 1.100 & 198.8 & 100.6 & 0.660 & 1.664 & 0.0130 & 0.296 & 2.694 \\
\hline
\end{tabular}

oscillation of the working gas temperature, which implies a sharper variation of the temperature gradient inside the thermal boundary layer. This thinking still needs to be validated by experimental observation or numerical modeling of the temperature distribution.

More results of the Nusselt number with diverse maximum Reynolds number and Valensi number under the mean working pressure of $2.0 \mathrm{MPa}$ are presented in Fig. 3. For different maximum Reynolds number and Valensi number, the Nusselt number always increases with a rise in the pressure ratio, and the difference lies in the slope of increase. The comparison of the square data and the circle data in Fig. 3 shows that a higher Valensi number leads to a larger increasing slope of the Nusselt number. Additionally, a higher maximum Reynolds number results in a smaller increasing slope of the Nusselt number, as presented by the circle data and the triangle data. The same situation can be seen from the experimental results under the mean working pressure of $2.5 \mathrm{MPa}$, as shown in Fig. 4. Thus, it can be concluded that the impact of compression-expansion effect on the heat transfer of the oscillating flow is more significant in the cases with a higher Valensi number and a lower maximum Reynolds number. The thermal boundary layer thickness, which can be denoted by the thermal penetration depth $\left(\delta_{\kappa}=(2 \alpha / \omega)^{0.5}\right.$, where $\alpha$ is the thermal diffusivity of the working gas), will become smaller in a higher Valensi number situation. The thinner thermal boundary layer may be more sensitive to the compression-expansion effect. In contrast, a higher maximum Reynolds number implies a stronger convection effect, which may relatively weaken the impact of the compression-expansion effect.

Figs. 3 and 4 also show that the increases in the maximum Reynolds number and in the Valensi number can both lead to a rise in the Nusselt number. That is, raising the maximum Reynolds number and the Valensi number can both enhance the heat transfer in the oscillating flow.

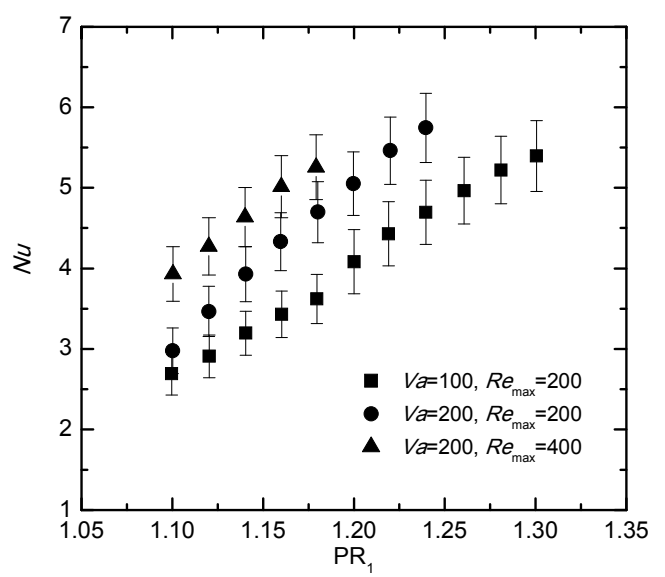

Fig. 3 Dependence of the Nusselt number on the pressure ratio with a mean working pressure of $2.0 \mathrm{MPa}$

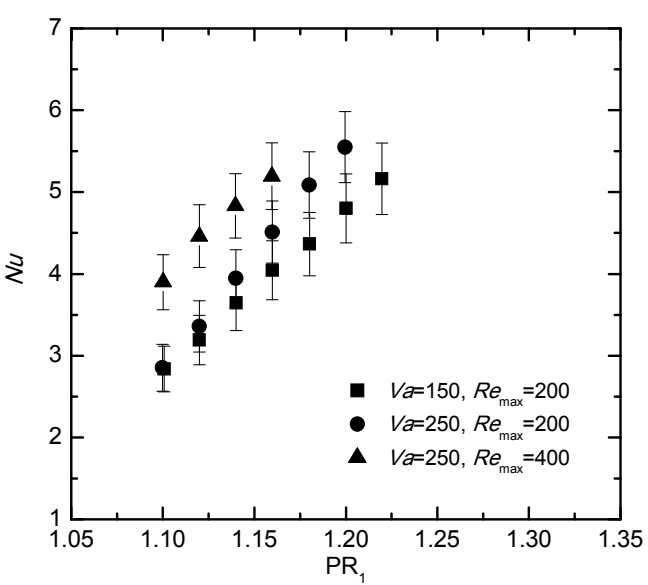

Fig. 4 Dependence of the Nusselt number on the pressure ratio with a mean working pressure of $2.5 \mathrm{MPa}$

\section{Conclusions}

An experimental apparatus has been built to test the heat transfer of a finned heat exchanger, aiming for the application of ambient-temperature heat 
exchangers in pulse tube refrigerators. The emphasis of the experimental observation was put on the impact of the compression-expansion effect, indicated by the pressure ratio, on the heat transfer of oscillating flow, which was seldom taken into account in previous studies. The experimental results indicate that the pressure ratio is an independent factor, and an increase in the pressure ratio can lead to a marked rise in the Nusselt number. The increase of the pressure ratio from 1.100 to 1.300 results in the rise of the Nusselt number from 2.694 to 5.395 with the maximum Reynolds number and the Valensi number fixed at 200 and 100, respectively. Moreover, the impact of the compression-expansion effect on the heat transfer in the oscillating flow becomes more significant for the cases with a higher Valensi number and a lower maximum Reynolds number. It is suggested that the pressure ratio should be considered in the treatment of the heat transfer of the oscillating flow.

\section{References}

Anderson, J.D., 1995. Computational Fluid Dynamics: The Basics with Applications. McGraw-Hill, New York.

Bouvier, P., Stouffs, P., Bardon, J.P., 2005. Experimental study of heat transfer in oscillatory flow. International Journal of Heat and Mass Transfer, 48(12):2473-2482. [doi:10. 1016/j.ijheatmasstransfer.2005.01.037]

Chen, Y., Luo, E.C., Dai, W., 2009. Heat transfer characteristics of oscillating flow regenerators in cryogenic temperature range below $20 \mathrm{~K}$. Cryogenics, 49(7):313-319.

Cooper, W.L., Nee, V.W., Yang, K.T., 1994. An experimental investigation of convective heat transfer from the heated floor of a rectangular duct to a low frequency, large tidal displacement oscillating flow. International Journal of Heat and Mass Transfer, 37(4):581-592.

de Waele, A.T.A.M., 2011. Basic operation of cryocoolers and related thermal machines. Journal of Low Temperature Physics, 164(5-6):179-236. [doi:10.1007/s10909-0110373-x]

de Waele, A.T.A.M., 2012. Finite heat-capacity effects in regenerators. Cryogenics, 52(1):1-7. [doi:10.1016/j. cryogenics.2011.09.015]

Dietrich, M., Thummes, G., 2010. Two-stage high frequency pulse tube cooler for refrigeration at $25 \mathrm{~K}$. Cryogenics, 50(4):281-286. [doi:10.1016/j.cryogenics.2010.01.010]

Gusev, V., Lotton, P., Bailliet, H., Job, S., Bruneau, M., 2001. Thermal wave harmonics generation in the hydrodynamical heat transport in thermoacoustics. Journal of the Acoustical Society of America, 109(1):84-90.

Hino, M., Sawamoto, M., Takasu, S., 1976. Experiments on transition to turbulence in an oscillatory pipe-flow. Journal of Fluid Mechanics, 75(2):193-207.
Incropera, F.P., DeWitt, D.P., Bergman, T.L., Lavine, A.S., 2007. Fundamentals of Heat and Mass Transfer, 6th Edition. Wiley, New York.

Jaworski, A.J., Piccolo, A., 2012. Heat transfer processes in parallel-plate heat exchangers of thermoacoustic devices -numerical and experimental approaches. Applied Thermal Engineering, 42:145-153.

Meng, F., Wang, M., Li, Z., 2008. Lattice Boltzmann simulations of conjugate heat transfer in high-frequency oscillating flows. International Journal of Heat and Fluid Flow, 29(4):1203-1210. [doi:10.1016/j.jheatfluidflow.2008.03. 001]

Merkli, P., Thomann, H., 1975. Transition to turbulence in oscillating pipe flow. Journal of Fluid Mechanics, 68(3):567-575.

Nsofor, E.C., Celik, S., Wang, X.D., 2007. Experimental study on the heat transfer at the heat exchanger of the thermoacoustic refrigerating system. Applied Thermal Engineering, 27(14-15):2435-2442. [doi:10.1016/j.applthermaleng.2007.03.008]

Piccolo, A., 2011. Numerical computation for parallel plate thermoacoustic heat exchangers in standing wave oscillatory flow. International Journal of Heat and Mass Transfer, 54(21-22):4518-4530. [doi:10.1016/j.ijheat masstransfer.2011.06.027]

Qiu, L.M., Zhi, X.Q., Han, L., Cao, Q., Gan, Z.H., 2012. Performance improvement of multi-stage pulse tube cryocoolers with a self-precooled pulse tube. Cryogenics, 52(10):575-579. [doi:10.1016/j.cryogenics.2012.05.002]

Shi, L., Yu, Z., Jaworski, A.J., 2010. Application of laser-based instrumentation for measurement of time-resolved temperature and velocity fields in the thermoacoustic system. International Journal of Thermal Sciences, 49(9):16881701. [doi:10.1016/j.ijthermalsci.2010.03.015]

Swift, G.W., 1992. Analysis and performance of a large thermoacoustic engine. Journal of the Acoustical Society of America, 92(3):1551-1563.

Swift, G.W., 2002. Thermoacoustics: A Unifying Perspective for Some Engines and Refrigerators. Acoustical Society of America, American Institute of Physics, New York.

Tang, K., Huang, Z.J., Jin, T., Bao, R., Chen, G.B., 2007. Influence of input acoustic power on regenerator's performance. Journal of Zhejiang University-SCIENCE A, 8(9):1452-1456. [doi:10.1631/jzus.2007.A1452]

Tang, K., Huang, Z.J., Jin, T., Chen, G.B., 2008. Impact of load impedance on the performance of a thermoacoustic system employing acoustic pressure amplifier. Journal of Zhejiang University-SCIENCE A, 9(1):79-87. [doi:10.1631/ jzus.A071340]

Tang, K., Zhang, Y., Lin, X.G., Jin, S.H., Jin, T., 2011. Hydrodynamic and thermal development of compressible oscillatory flow inside circular channel. Cryogenics, 51(3):139-145. [doi:10.1016/j.cryogenics.2010.12.011]

Zhao, T.S., Cheng, P., 1996. Oscillatory heat transfer in a pipe subjected to a laminar reciprocating flow. Journal of Heat Transfer-Transactions of the ASME, 118(3):592-597. 


\section{Appendix}

This appendix presents the determination of the five dimensionless similarity criteria, i.e., the maximum Reynolds number $R e_{\max }$, the Valensi number $V a$, the Prandtl number $P r$, the specific heat ratio $\gamma$, and the Mach number $M a$.

The governing equations for the unsteady flow of compressible viscous fluid, as well as the equation of state for ideal gas, are as follows (Anderson, 1995):

$$
\begin{aligned}
& \frac{\partial \rho}{\partial t}+\nabla \cdot(\rho \boldsymbol{V})=0, \\
& \rho \frac{\partial \boldsymbol{V}}{\partial t}+\nabla \boldsymbol{V} \cdot(\rho \boldsymbol{V})=-\nabla p-\frac{2}{3} \nabla[\mu(\nabla \cdot \boldsymbol{V})] \\
& +\nabla \cdot\left[\mu(\nabla \boldsymbol{V})^{\mathrm{T}}\right]+\nabla \cdot[\mu(\nabla \boldsymbol{V})] g, \\
& \rho c_{\mathrm{p}} \frac{\partial T}{\partial t}+\rho c_{\mathrm{p}} \boldsymbol{V} \cdot \nabla T \\
& =\nabla \cdot[k(\nabla T)]+\left[\frac{\partial p}{\partial t}+\boldsymbol{V} \cdot \nabla p\right]+\Phi, \\
& p=\rho R T,
\end{aligned}
$$

where $T, p, \rho, c_{\mathrm{p}}, \mu, k$, and $R$ are the temperature, pressure, density, constant-pressure specific heat, viscosity, thermal conductivity, and gas constant, respectively. $\boldsymbol{V}$ is the vector of velocity, $(\nabla \cdot \boldsymbol{V})$ and $(\nabla \boldsymbol{V})^{\mathrm{T}}$ are two tensors, $t$ is the time, and $\Phi$ denotes the energy dissipation due to the viscosity.

The following dimensionless parameters with the superscript ${ }^{*}$ are defined to normalize Eqs. (A1)(A4).

$$
\left\{\begin{array}{l}
x^{*}=\frac{x}{L_{0}}, y^{*}=\frac{y}{L_{0}}, \\
z^{*}=\frac{z}{L_{0}}, t^{*}=\frac{t}{t_{0}}, \\
\boldsymbol{V}^{*}=\frac{\boldsymbol{V}}{u_{0}}, T^{*}=\frac{T}{T_{0}}, \\
p^{*}=\frac{p}{p_{0}}, \rho^{*}=\frac{\rho}{\rho_{0}}, \\
c_{\mathrm{p}}^{*}=\frac{c_{\mathrm{p}}}{c_{\mathrm{p} 0}}, \mu^{*}=\frac{\mu}{\mu_{0}}, \\
k^{*}=\frac{k}{k_{0}}, \Phi^{*}=\frac{\Phi}{\mu_{0}\left(u_{0} / L_{0}\right)^{2}},
\end{array}\right.
$$

where the subscript 0 indicates the characteristic parameters to nondimensionalize the variables.

Substituting Eq. (A5) into Eqs. (A1)-(A4) gives the nondimensional forms of the governing equations and the equation of state as follows:

$$
\begin{aligned}
& \frac{\partial \rho^{*}}{\partial t^{*}}+\frac{t_{0} u_{0}}{L_{0}} \nabla \cdot\left(\rho^{*} \boldsymbol{V}^{*}\right)=0, \\
& \rho^{*} \frac{\partial \boldsymbol{V}^{*}}{\partial t^{*}}+\frac{t_{0} u_{0}}{L_{0}} \nabla \boldsymbol{V}^{*} \cdot\left(\rho^{*} \boldsymbol{V}^{*}\right) \\
& =-\frac{t_{0} R T_{0}}{u_{0} L_{0}} \nabla p^{*}-\frac{2}{3} \frac{t_{0} v_{0}}{L_{0}^{2}} \nabla\left[\mu^{*}\left(\nabla \cdot \boldsymbol{V}^{*}\right)\right] \\
& \quad+\frac{t_{0} v_{0}}{L_{0}^{2}} \nabla \cdot\left[\mu^{*}\left(\nabla \boldsymbol{V}^{*}\right)^{\mathrm{T}}\right]+\frac{t_{0} v_{0}}{L_{0}^{2}} \nabla \cdot\left[\mu^{*}\left(\nabla \boldsymbol{V}^{*}\right)\right], \\
& \rho^{*} c_{\mathrm{p}}^{*} \frac{\partial T^{*}}{\partial t^{*}}+\frac{t_{0} u_{0}}{L_{0}} \rho^{*} c_{\mathrm{p}}^{*} \boldsymbol{V} \cdot \nabla T^{*} \\
& =\frac{t_{0} \alpha_{0}}{L_{0}{ }^{2}} \nabla \cdot\left[k^{*}\left(\nabla T^{*}\right)\right] \\
& \quad+\left[\frac{R}{c_{\mathrm{p} 0}} \frac{\partial p^{*}}{\partial t^{*}}+\frac{R t_{0} u_{0}}{c_{\mathrm{p} 0} L_{0}} \boldsymbol{V}^{*} \cdot \nabla p^{*}\right]+\frac{v_{0} t_{0} u_{0}^{2}}{c_{\mathrm{p} 0} T_{0} L_{0}^{2}} \Phi^{*}, \\
& p^{*}=\rho^{*} T^{*},
\end{aligned}
$$

where $v$ and $\alpha$ are the kinematic viscosity and the thermal diffusivity, respectively.

Considering the characteristics of the oscillating flow, we set

$$
\left\{\begin{array}{l}
L_{0}=D_{\mathrm{h}}, \\
t_{0}=\frac{1}{\omega}, \\
u_{0}=u_{\mathrm{A}},
\end{array}\right.
$$

and

$$
\left\{\begin{array}{l}
R e_{\text {max }}=\frac{u_{0} L_{0}}{v_{0}}=\frac{u_{\mathrm{A}} D_{\mathrm{h}}}{v_{0}}, \\
V a=\frac{L_{0}^{2}}{t_{0} v_{0}}=\frac{\omega D_{\mathrm{h}}^{2}}{v}, \\
\gamma=\frac{c_{\mathrm{p} 0}}{c_{\mathrm{v} 0}}, \\
P r=\frac{v_{0}}{\alpha_{0}}, \\
M a=\frac{u_{0}}{a_{0}}=\frac{u_{\mathrm{A}}}{a_{0}} .
\end{array}\right.
$$


With Eq. (A11), Eqs. (A6)-(A8) can be rewritten as

$$
\begin{aligned}
& \frac{\partial \rho^{*}}{\partial t^{*}}+\frac{R e_{\max }}{V a} \nabla \cdot\left(\rho^{*} \boldsymbol{V}^{*}\right)=0, \\
& \rho^{*} \frac{\partial \boldsymbol{V}^{*}}{\partial t^{*}}+\frac{R e_{\max }}{V a} \nabla \boldsymbol{V}^{*} \cdot\left(\rho^{*} \boldsymbol{V}^{*}\right) \\
& =-\frac{R e_{\max }}{\gamma V M a^{2}} \nabla p^{*}-\frac{2}{3} \frac{1}{V a} \nabla\left[\mu^{*}\left(\nabla \cdot \boldsymbol{V}^{*}\right)\right] \\
& \quad+\frac{1}{V a} \nabla \cdot\left[\mu^{*}\left(\nabla \boldsymbol{V}^{*}\right)^{\mathrm{T}}\right]+\frac{1}{V a} \nabla \cdot\left[\mu^{*}\left(\nabla \boldsymbol{V}^{*}\right)\right], \\
& \rho^{*} c_{\mathrm{p}}^{*} \frac{\partial T^{*}}{\partial t^{*}}+\frac{R e_{\max }}{V a} \rho^{*} c_{\mathrm{p}}^{*} \boldsymbol{V}^{*} \cdot \nabla T^{*}
\end{aligned}
$$

Eqs. (A12)-(A14), we can determine the five dimensionless similarity criteria for the oscillating flow, i.e., the maximum Reynolds number $R e_{\max }$, the Valensi number $\mathrm{Va}$, the Prandtl number $P r$, the specific heat ratio $\gamma$, and the Mach number $M a$.

\section{Recommended paper related to this topic}

\section{Lumped parameter model for resonant frequency estimation of a thermoacoustic engine with gas-liquid coupling oscillation}

Authors: Ke Tang, Tian Lei, Xiao-gang Lin, Tao Jin, Yu Zhang

Journal of Zhejiang University-SCIENCE A (Applied Physics \& Engineering), 2011, Vol. 12, No. 3, P.232-237

doi:10.1631/jzus.A1000191

Abstract: Gas-liquid coupling oscillation is a novel approach to reducing the resonant frequency and to elevating the pressure amplitude of a thermoacoustic engine. If a thermoacoustic engine is used to drive low-frequency pulse tube refrigerators, the frequency matching between the thermoacoustic engine and the refrigerator plays an important role. Based on an acoustic-electric analogy, a lumped parameter model is proposed to estimate the resonant frequency of a standing-wave thermoacoustic engine with gas-liquid coupling oscillation. Furthermore, a simplified lumped parameter model is also developed to reduce the computation complexity. The resonant frequency dependence on the mean pressure, the gas space volume, and the water column length is computed and analyzed. The impact of different working gases on the resonant frequency is also discussed. The effectiveness of the models is validated by comparing the computed results with the experimental data of the gas-liquid coupling oscillation system. An increase in the mean working pressure can lead to a rise in the resonant frequency, and a lower resonant frequency can be achieved by elongating the liquid column. In comparison with nitrogen and argon, carbon dioxide can realize a lower frequency due to a smaller specific heat ratio. 